\title{
Representações sociais da morte para pessoas que vivem com HIV/AIDS
}

\author{
Social representations of death held by people living with HIV/AIDS \\ Representaciones sociales de la muerte para personas que viven con $\mathrm{VIH} / \mathrm{SIDA}$
}

\author{
Antonio Marcos Tosoli Gomes'; Ana Dulce Santana dos Santos"'; Sérgio Corrêa Marques'"l, Virgínia Paiva Figueiredo

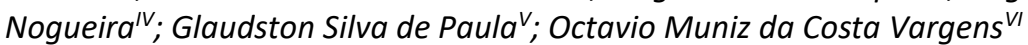

\begin{abstract}
RESUMO
Objetivo: descrever os conteúdos e a organização das representações sociais da morte para pessoas que vivem com HIV/AIDS Método: estudo descritivo, de abordagem qualitativa, e delineado por meio da Teoria das Representações Sociais. Participaram 165 pessoas vivendo com HIV/AIDS, em tratamento no ambulatório de referência na cidade do Rio de Janeiro. Projeto aprovado por Comitê de Ética em Pesquisa. Utilizou-se o software Evoc, ao final construiu-se o quadro de quatro casas. Resultados: abalizam uma dimensão emocional e do conhecimento das representações da morte, como um objeto multifacetado com diversos significados e variados sentimentos, apontando para a naturalização da morte. Conclusão: ainda que a morte contenha uma representação negativa, em pessoas com HIV/AIDS, pelos sentimentos ligados à tristeza, medo e dor, as estratégias políticas e governamentais, medidas de prevenção e tratamento no controle da doença delineiam uma nova direção, diante da cronicidade da síndrome, resultando em representações imbricadas no sentido da vida, do viver e do morrer como processo natural.
\end{abstract}

Descritores: Enfermagem; HIV; síndrome da imunodeficiência adquirida; morte.

\section{ABSTRACT}

Objective: to describe the content and organization of social representations of death in people living with HIV/AIDS. Method: in this qualitative, descriptive study based on Social Representation Theory, the participants were 165 people living with HIV/AIDS, in treatment at an referral outpatient clinic in Rio de Janeiro city. The research ethics committee approved the study. A four-box chart was set up using Evoc software. Results: indicate an emotional dimension and knowledge of representations of death as a multifaceted object with a diverse meanings and varied feelings, pointing to a naturalization of death. Conclusion: even though death does contain negative representations for people with HIV/AIDS, due to feelings of sadness, fear and pain, government policy strategies and prevention and treatment measures for controlling the disease point in new directions, in view of the syndrome's timeframe, resulting in representations that overlap in the meaning of life, of living and of dying as a natural process.

Descriptors: Nursing; HIV; acquired immunodeficiency syndrome; death.

\section{RESUMEN}

Objetivo: describir los contenidos y la organización de las representaciones sociales de la muerte respecto a las personas que viven con VIH-SIDA. Método: estudio descriptivo, con enfoque cualitativo, basado en la teoría de las representaciones sociales. Participaron 165 personas, que viven con VIH-SIDA, en tratamiento en una clínica ambulatoria de referencia en Río de Janeiro. El proyecto fue aprobado por el Comité de Ética. Usando el software Evoc, se construyó un marco de cuatro elementos. Resultados: indican una dimensión emocional y el conocimiento de las representaciones de la muerte como un objeto multifacético con varios significados y sentimientos, que apuntan hacia la naturalización de la muerte. Conclusión: aunque la muerte contenga una representación negativa para las personas con VIH-SIDA, debido a sentimientos relacionados con la tristeza, el miedo y el dolor, las estrategias políticas y gubernamentales, medidas de prevención y tratamiento en el control de la enfermedad delinean una nueva dirección, ante la cronicidad del síndrome, que condujo a representaciones imbricadas en el sentido de la vida, de vivir y de morir como un proceso natural.

Descriptores: Enfermería; VIH; síndrome de inmunodeficiencia adquirida; muerte.

\section{INTRODUÇÃO}

A morte faz parte do desenvolvimento humano significando e ressignificando a vida ${ }^{1}$. As pessoas que vivem com o vírus da imunodeficiência humana (HIV) e a síndrome da imunodeficiência adquirida (AIDS), por muito tempo, apresentaram suas representações alicerçadas no imaginário coletivo da AIDS como sentença de morte. A estruturação das representações se apoia em crenças apontando os aspectos e sentimentos negativos para a síndrome que repercutiam como sinônimo de morte ${ }^{2}$.

\footnotetext{
IEnfermeiro. Doutor em Enfermagem. Professor Titular, Universidade do Estado do Rio de Janeiro. Brasil. E-mail: mtosoli@gmail.com.

"Enfermeira. Doutoranda em Enfermagem, Doutorado Interinstitucional. Universidade do Estado do Rio de Janeiro. Brasil. E-mail: anadulcesantana@yahoo.com.br. "'Enfermeiro Doutor em Enfermagem. Professor Adjunto, Universidade do Estado do Rio de Janeiro. Brasil. E-mail: sergiocmarques@uol.com.br.

IVEnfermeira. Doutoranda em Enfermagem, Universidade do Estado do Rio de Janeiro. Brasil. E-mail: virginiafigueiredo@yahoo.com.br.

vEnfermeiro. Doutorando em Enfermagem. Universidade do Estado do Rio de Janeiro. Brasil. E-mail: glaudstondepaula@gmail.com.

v'Enfermeiro Obstetra. Doutor em Enfermagem. Professor Titular, Universidade do Estado do Rio de Janeiro. Brasil. E-mail: omcvargens@uol.com.br.

VIIAgradecimento ao Enfermeiro Doutor Érick Igor dos Santos, in memoriam, pela colaboração nesta pesquisa e por sua breve e intensa vida dedicada a enfermagem.
} 
Entretanto, os avanços no tratamento da infecção, com a incorporação da terapia antirretroviral em associação à prevenção, resultaram em importantes melhorias relativas à sobrevida de pessoas vivendo com HIV, derivando na redução da morbimortalidade ${ }^{2-4}$.

A redução da mortalidade por AIDS no país, com taxas ainda que equilibradas nacionalmente, mostra progresso importante em alguns estados brasileiros. Nos últimos 10 anos no Brasil passou-se de 6,0 óbitos a cada 100 mil habitantes em 2005 para 5,7 em 2014, o que representa uma queda de 5\% ${ }^{5}$. E, embora seja um problema de saúde mundial, em muitos países o número de novas infecções pelo HIV continua a diminuir, com declínio de $75 \%$ em 10 países e por mais de 50\% em 27 países; na América Latina houve um declínio de novas infecções de 3\% entre 2005 e $2013^{6}$. A queda na mortalidade favorece a transferência de expectativas negativas por positivas, à medida que a AIDS passa da condição de doença aguda e fatal, sinônimo de morte e, passe a ser entendida como doença crônica e como tal evolui como processo natural ${ }^{7}$.

Diante do exposto, traçou-se como objetivo, neste estudo, descrever os conteúdos e a organização das representações sociais da morte para pessoas que vivem com HIV.

\section{REVISÃO DE LITERATURA}

A morte é um fenômeno biológico que integra o ciclo natural da vida, e aponta a finitude do ser humano, o que assinala os aspectos intrapsíquicos ${ }^{1}$. Pois, a morte é uma questão apenas para o humano, por isso tem sido objeto de produção artística, de poetas, músicos, romanceada com grandes trabalhos; na produção dos pintores ilustres que deixaram seus registros sobre a maneira de se ver a morte ${ }^{1,8}$. Importante considerá-la como fenômeno não só biológico, mas individual, bem como, social, cujos aspectos psicológicos, filosóficos, religiosos, éticos, históricos, culturais e jurídicos devem ser considerados na medida em que são fundamentais para que o ser humano consiga enfrentar com dignidade o seu limite angustiante ${ }^{2}$.

Morrer, assim como nascer, é uma consequência natural da vida, pelo qual todos os seres humanos terão de passar um dia ${ }^{8}$. E, ainda para o homem, é muito difícil internalizar e conviver com o sentimento da própria finitude ${ }^{8,9}$. $\mathrm{E}$ o olhar para a AIDS, leva-o a refletir sobre a morte, e isto pode configurar sua impotência no controle da sua vida ${ }^{1}$, colocando-o diante do incompleto e do não terminado.

Na sociedade ocidental, não só evita-se falar, conviver e pensar na morte como algo natural, mas, sobretudo, evita-se conversar sobre o processo de morrer e as causas da morte ${ }^{1,2,8-10}$. Portanto, pode-se considerar o medo da morte como o medo do desconhecido e o desamparo no final da vida ${ }^{11}$. A consciência da morte faz com que a vida importe, pois, sua possibilidade transforma a maneira como se vive a vida ${ }^{12}$. Assim, caminha-se, acolhendo a morte como processo natural da existência.

\section{MÉTODO}

Trata-se de um estudo descritivo, de abordagem qualitativa e delineada por meio da Teoria das Representações Sociais em sua abordagem estrutural ${ }^{3,7,13-17}$. A pesquisa foi realizada no período compreendido entre março e outubro de 2015 em uma instituição pública, referência para tratamento ambulatorial às pessoas que vivem com HIV, localizada no município do Rio de Janeiro/RJ, Brasil.

Os dados sociodemográficos para caracterização do grupo foram coletados por questionário e os conteúdos da representação por meio da técnica de evocações livres ao termo indutor morte. Vale registrar que a caracterização sociodemográfica dos participantes não é analisada no presente estudo.

A amostra por conveniência foi definida considerando a totalidade de usuários atendidos na referida unidade, sem perder de vista o número mínimo de sujeitos necessários para a recuperação de representações na abordagem estrutural da teoria ${ }^{3,7,13-15}$. Logo, participaram, efetivamente, 165 pessoas vivendo com HIV, tendo como critérios de inclusão - idade maior que 18 anos, sem faixa-etária limítrofe e com no mínimo 6 meses de diagnóstico. 0 critério de exclusão proposto considerou a presença de limitações cognitivas ou de comunicação que inviabilizassem a coleta de dados.

Em observância às determinações da Resolução $n^{\circ} 466$ de 12 de dezembro de 2012, do Conselho Nacional de Saúde/Ministério da Saúde, o projeto do estudo foi submetido ao Comitê de Ética em Pesquisa com Seres Humanos (CEPq) da Universidade do Estado do Rio de Janeiro (UERJ), sendo aprovado pelo parecer número 699.220.

Os dados provenientes do questionário foram registrados em planilha do software Excel e analisados pela estatística descritiva. Os conteúdos oriundos da evocação livre foram analisados pela técnica do Quadro de Quatro Casas, com o emprego do software Ensemble des programmes permettant l'analyse de evocations (EVOC), versão 2005. 
O software calculou e informou a frequência simples de ocorrência de cada palavra evocada, a média de ocorrência de cada palavra por ordem de evocação e a média das ordens médias ponderadas do conjunto dos termos evocados ${ }^{3,7,16}$.

Ao final, foi produzido o quadro de quatro casas onde os termos localizados no quadrante superior esquerdo correspondem aos possíveis elementos centrais da representação, compreendendo os conteúdos provavelmente mais significativos sob a ótica dos participantes do estudo. Os elementos localizados no quadrante inferior esquerdo constituem a zona de contraste da representação, que são elementos de baixa frequência e baixa ordem média de evocação, ou seja, são prontamente evocados. Já no quadrante superior direito estão os elementos que possuem alta frequência, apesar de serem menos prontamente evocados. No quadrante inferior direito, estão os elementos de segunda periferia, menos frequentes e menos prontamente evocados, que são os elementos mais periféricos e mais afastados do núcleo da representação ${ }^{3,7}$.

Segundo a Teoria do Núcleo Central (TNC), o quadro de quatro casas divide-se em dois sistemas distintos: um central e outro periférico. O primeiro atribui estabilidade, organização e sentido à representação identificada e o sistema periférico é flexível, integra novas informações à estrutura da representação, protege o núcleo central e está mais voltado à prática e a situações concretas. Os termos que atendem, ao mesmo tempo, aos critérios de maior frequência e mais prontamente evocados possuem maior importância no pensamento social dos participantes, sendo os prováveis integrantes do núcleo central da representação ${ }^{3}$.

Após a finalização desta técnica, procedeu-se à análise de similitude dos conteúdos presentes no quadro de quatro casas, com o objetivo de identificar o grau de conexidade entre eles visando dar mais uma indicação da centralidade de determinados elementos.

\section{RESULTADOS}

\section{A estrutura representacional da morte para pessoas vivendo com HIV}

Para o termo morte foram evocadas 645 palavras, entre as quais 244 eram diferentes. A frequência mínima definida foi de 8 , sendo excluídas palavras com frequência menor do que esta. A frequência média calculada dos termos restantes foi de 14, com base na Lei de Zipf. A ordem média de evocação (OME) foi de 2,6 em uma escala de 1 a 5 . A partir destes parâmetros foi constituído o quadro de quatro casas, evidenciado no Figura 1.

\begin{tabular}{|c|l|c|c|l|c|c|}
\hline O.M.E. & \multicolumn{3}{|c|}{$<$} & \multicolumn{3}{c|}{$\geq \mathbf{2 , 6}$} \\
\hline $\begin{array}{l}\text { Freq. } \\
\text { Med. }\end{array}$ & Termo evocado & Freq. & O.M.E. & Termo evocado & Freq. & O.M.E. \\
\hline \multirow{3}{*}{$\geq \mathbf{1 4}$} & tristeza & 32 & 2,563 & outra vida & 21 & 2,667 \\
& medo & 28 & 1,929 & descanso & 20 & 2,600 \\
& fim & 21 & 2,333 & & & \\
& & & & & & \\
\hline \multirow{3}{*}{} & natural & 13 & 1,846 & Deus & 13 & 3,308 \\
& não medo & 13 & 1,769 & saudade & 13 & 2,846 \\
& passagem & 13 & 2,154 & dor & 12 & 2,917 \\
& perda & 12 & 2,167 & paz & 11 & 2,727 \\
& sofrimento & 10 & 2,500 & recomeço & 10 & 2,900 \\
& missão & 8 & 2,500 & acabou & 10 & 2,700 \\
& & & & aceitação & 10 & 3,100 \\
\hline
\end{tabular}

FIGURA 1: Quadro de quatro casas ao termo indutor morte. Município do Rio de Janeiro, Brasil, 2015.

O quadrante superior esquerdo é formado pelos elementos possivelmente centrais, que neste estudo são: tristeza, medo e fim, que indicam forte dimensão emocional e do conhecimento, apresentando, portanto, um caráter normativo. No contexto da TNC, o caráter normativo está ligado ao sistema de valores do grupo investigado; já o caráter funcional privilegia na representação e na constituição do núcleo central elementos mais importantes para a realização e justificação de uma tarefa, ou seja, elementos ligados a uma ação, que não são evidenciados neste quadrante 7,15 .

As palavras tristeza e medo mostram-se possivelmente oriundas da entidade mórbida da AIDS em sua repercussão física mais extrema, qual seja a morte. A morte, no contexto da AIDS, mostra-se ameaçadora e, por isso, desperta sentimentos negativos no grupo social. Dessa forma, apesar da atitude negativa por parte do grupo frente ao objeto 
morte, a palavra fim enuncia uma tentativa de aproximação conceitual ao objeto de representação para incorporá-la ao universo psicossocial dos participantes. Essa dimensão emocional, assim, visa racionalizar a dimensão emocional apontada anteriormente para assentá-la na subjetividade do grupo social, provocando, dessa maneira, uma tensão entre as duas dimensões representacionais.

É importante ressaltar que as palavras que compõem o provável núcleo central possuem frequência bastante superior ao ponto de corte calculado, qual seja o de 14. Esta característica aponta para alta recorrência dessas palavras entre os integrantes do grupo quando solicitados que evocassem as cinco primeiras palavras ou expressões que lhes ocorressem após ouvirem o termo indutor morte. Reforça a ideia de dimensão emocional presente na representação da morte para pessoas que vivem com HIV. Nesse contexto, o termo medo é o que possui a menor OME se destacando como uma das palavras mais prontamente evocadas de toda a estrutura representacional.

O quadrante inferior esquerdo, também denominado zona de contraste, é o segundo mais importante na estrutura da representação segundo a TNC, reforçando os elementos centrais ou indicando a presença de um subgrupo. Nesse quadrante foram identificadas as palavras natural, não-medo, passagem, perda, sofrimento e missão. Os termos perda e sofrimento parecem reforçar a dimensão emocional da representação em seu provável núcleo central. Por outro lado, os termos natural, passagem e missão sugerem o reforço da dimensão do conhecimento anteriormente destacada no provável núcleo central da representação e a existência de um esforço psicossocial do grupo para apropriar-se de um objeto altamente abstrato como a morte. Nesse sentido, face à ameaça da morte em seu viver cotidiano com a soropositividade ou o desenvolvimento da síndrome, os participantes do estudo sofrem pressão à inferência, percebendo-se na incumbência de ressignificar a morte para melhor conviver com a ideia de sua proximidade.

Ainda na zona de contraste, a expressão não-medo pode estar atrelada a um possível subgrupo cuja representação da morte destoa do grupo geral.

Na primeira periferia, localizada no quadrante superior direito, encontram-se as evocações outra-vida e descanso, que reforçam a inferência de certa racionalidade na estrutura representacional. Observa-se que o sentido da morte, para o grupo investigado, pode estar atrelado a uma outra existência além da vida presente, existência esta capaz de promover repouso às atribulações do viver com HIV. Esta inferência ganha força em virtude da evocação passagem na zona de contraste, o que reitera o caráter processual da morte para o grupo investigado.

No quadrante inferior direito, ou segunda periferia, estão reunidas as palavras ou expressões não tão importantes para o grupo sobre o objeto de representação ou, então, que estão refletindo o contexto de vida desse grupo. Nele estão os termos Deus, saudade, dor, paz, recomeço, acabou e aceitação. O termo Deus expressa uma dimensão imagética da representação pautada na figura divina. Os termos saudade e dor reforçam a afetividade do provável núcleo central, justificando o evocar dos termos medo e tristeza no quadrante superior esquerdo. Paz, recomeço e acabou expressam os produtos da finitude da vida para o grupo e estão imbricados na racionalização do processo de morrer. Aceitação enuncia uma dimensão prática da representação que, mesmo situando-se mais perifericamente na estrutura representacional, leva a crer que os participantes estão, de fato, sob forte pressão à inferência e, assim, sentem-se na incumbência de aceitar o processo futuro da morte em face de sua inevitabilidade.

Percebe-se, na estrutura representacional, que o sistema central possui maior teor negativo enquanto que os elementos de teor positivo existentes compõem mais o sistema periférico da representação.

\section{Análise da conexidade dos elementos da representação social da morte para pessoas que vivem com HIV/AIDS}

Em determinados contextos, alguns dos elementos que compõem o sistema periférico podem possuir centralidade em virtude de suas altas frequências. Para refutar ou reiterar esta hipótese, procedeu-se à análise de similitude dos termos que compuseram a estrutura representacional, conforme mostra a Figura 2.

A cognição que mais estabeleceu conexões na árvore máxima foi tristeza, já apontada como central no quadro de quatro casas, seguida de descanso, que, na análise estrutural, se encontra na primeira periferia. Em função da possibilidade de a conexidade ser considerada como um critério para a indicação de centralidade ${ }^{16}$, esses léxicos podem ser considerados, por hipótese, como pertencentes ao núcleo central da representação.

Nota-se que a cognição tristeza mantém conexão com mais elementos negativos, como medo, perda, dor, saudade, fim e sofrimento, característica que confirma o teor negativo da representação. Descanso, por outro lado, mostra alto índice de similitude com as cognições passagem, missão, paz e fim, o que indica que este é o sentido atribuído à morte pelas pessoas que vivem com HIV, na dimensão conhecimento da representação. 


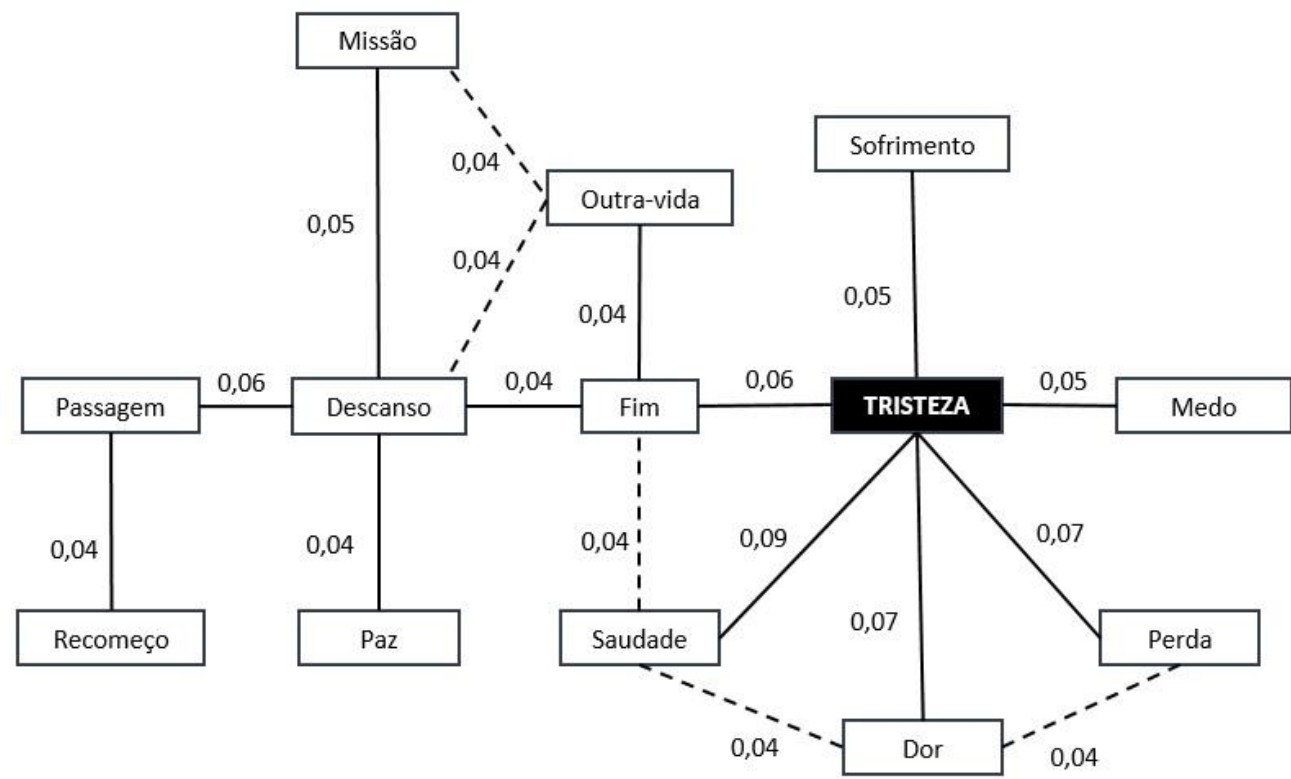

FIGURA 2: Árvore máxima da análise de similitude das representações sociais da morte para pessoas que vivem com HIV/AIDS. Rio de Janeiro, Brasil, 2015.

\section{DISCUSSÃO}

O estudo aponta como as prováveis cognições integrantes do núcleo central da representação os termos tristeza e descanso que, respectivamente, constituem uma dimensão emocional e do conhecimento, refletidas em todos os quadrantes. Os participantes constroem representações e conceito de morte pela experiência vivenciada e compartilhada ao longo de suas vidas. Para quem vive com HIV, mesmo com o progresso científico na batalha contra o vírus, o aparecimento dos sintomas e o agravamento da doença, agregados à perspectiva da morte, refletem intensamente nas suas expectativas de vidas ${ }^{1-2}$.

As palavras descanso e fim expressam os mesmos sentidos, a partir da análise estrutural, e numa tentativa de aproximação conceitual do grupo aqui investigado, estão condicionadas pelas próprias representações sociais ancoradas historicamente na construção social da AIDS, que traz, desde a sua origem, a relação com o sofrimento e a finitude ${ }^{18}$.

A morte, neste estudo, encontra-se representada como objeto multifacetado forte e intenso que precisa de várias definições e, estando, assim, atrelado a diversas emoções. E embora tenha uma representação predominantemente negativa, a evocação dos termos não-medo e natural, neste estudo, apontam a naturalização do processo de morte como algo próprio do ser humano, também representada de forma positiva de se significar o fenômeno. Estes achados, também, permitem inferir a ocorrência de um processo de mudança na representação da AIDS, pelo sentido mais positivo trazido no deslocamento desse elemento de sua centralidade. A infecção pelo HIV não mais representa uma sentença de morte, pois, atualmente é possível viver de forma saudável e assintomática por muitos anos ${ }^{2,3}$.

Nessa perspectiva, da morte como objeto multifacetado, destaca-se a dimensão do conhecimento nos termos natural, passagem e missão; destarte, seu potencial gerador e organizador na representação, descritas em torno desta cognição altamente abstrata. A morte é uma ocorrência não facilmente descrita e depende de como cada um a apreende. Porquanto, a questão transcende os aspectos naturais e biológicos, o que torna difícil um único termo que a possa traduzir, e está implicada com as experiências/vivenciadas por cada individuo ${ }^{2,9}$.

Os elementos que se apresentam estão ancorados com a crença ligada à concepção da AIDS que, anteriormente, no imaginário popular coletivo, estava associada à morte. As representações sobre AIDS são reproduzidas ao longo do processo sócio-histórico, destes mais de 30 anos de doença e, perpetuado através da comunicação no dia a dia dos indivíduos.

As cognições descanso, outra vida e passagem, que se encontram na periferia da estrutura da representação, reforçam o termo fim, presente no provável núcleo central, evidenciando formas mais positivas de significar a morte, refletindo uma dimensão mais espiritual, e por sua vez a qualifica como natural, portanto, sem provocar o medo (nãomedo), e sim o recomeço, que apontam para a crença da morte como missão, no sentido de traduzir a vida como um aprender a viver ${ }^{19}$. 
A alteração da forma de enfrentamento da morte, evocada como natural afasta a ideia de fracasso, e parece ser um dos elementos que embora se estabeleça no sistema periférico destas representações, já direciona para a estruturação da morte em pessoas com HIV, na própria evocação de aceitação da finitude biológica da existência humana. A morte não é mais considerada fracasso, pois ela faz parte da vida, é um acontecimento que se tem de viver ${ }^{20}$.

Estes achados, também, permitem inferir a ocorrência de um processo de mudança na representação da AIDS, pelo sentido mais positivo trazido pelo deslocamento desse elemento provável de sua centralidade ${ }^{3}$. Tal mudança se daria, sobretudo, pelo alinhamento da cognição morte dos elementos organizadores dessas representações sociais no cotidiano dos participantes deste estudo, em que, os sistemas periféricos despontam para a introdução de outras representação da morte, revelada pela estabilidade e melhor controle da doença, que sai da sua condição de aguda e fatal, para a crônica e, como tal naturalizada, com o seguimento da vida ${ }^{14}$. Apresenta-se, então, nas suas multifaces nominadas como recomeço, paz e passagem.

A dimensão emocional esteve presente em todos os quadrantes, e nos sentimentos sobre morte, a cognição tristeza é a que mantém mais conexões com outros termos, sendo todos de caráter negativo, como medo, perda, dor, sofrimento e saudade, configurando nesta particularidade parte do teor negativo da representação. O adoecer pela AIDS pode trazer para o homem a consciência da sua finitude, gerando sofrimento e dor. Entretanto, estes sentimentos de sofrimento e perda são atribuídos ao fato de o homem não aceitar a morte seu desafio é tentar vencê-la, buscando a imortalidade $\mathrm{e}^{1,9,20}$.

A morte é vista como impregnada de emoções ligados à tristeza, solidão e dor 1,2,4,21, representa um elemento carregado de sofrimento psíquico, da pessoa vivendo com HIV, sobretudo pelo aspecto cronológico.

Além do tratamento com antirretrovirais, a sociedade atual vem desenvolvendo estratégias no enfretamento, reunindo não só as pessoas vivendo com HIV, mas a sociedade civil nas suas diversas representações que se mobilizam, e se fazem representar para o acesso universal à prevenção, ao tratamento, à atenção e ao apoio, no combate ao HIV22.

As atitudes dos homens diante da morte são reflexos da sociedade, da temporalidade e da cultura das quais fazem parte ${ }^{22}$. Nesse sentido, os sentimentos negativos referentes à morte apresentados pelos homens podem estar imbricados pelo sistema cultural em que estão inseridos ${ }^{10}$.

Na sociedade ocidental, não só, evita-se de falar, de conviver e de pensar na morte como algo natural, mas, sobretudo, evita-se bastante falar no processo de morrer e nas causas da morte $1,2,8,10-12$.

O medo da morte, apesar de ser um sentimento comum nas pessoas, se acentua em alguns indivíduos ao se depararem com a doença. Há uma espécie de angústia diante da certeza da morte, um medo de morrer crescente ao se deparar com a doença e suas consequências ${ }^{2,23}$.

A relação entre esses termos sugere que o elemento morte da pessoa vivendo com HIV estaria relacionado ao fim e ressignifica-o, como clareza da finitude do homem, no encerramento da vida, pois todos irão morrer. Haja vista, o morrer é fato incontestável de todo ser humano, significando a morte como estágio final do crescimento humano ${ }^{21}$, trazendo representações em que a concepção espiritual ou filosófica signifique a morte como parte da existência do homem ${ }^{14}$.

O enfretamento da morte, que mobiliza tantos afetos e significados, remete à compreensão dos elementos que a define. A partir das representações sociais dos participantes desta pesquisa, com seus diversos significados e variados sentimentos, ligados à tristeza sofrimento, dor e medo, embora apresentem a ideia de fim, na descrição em descanso, passagem, paz, não-medo e natural, apontam para naturalização da morte em pessoas com HIV e, como ser humano, que vai morrer, ressignificando a vida a partir da consciência da sua morte ${ }^{24}$.

Verifica-se a necessidade de abordagens dialógicas grupais e individuais na promoção e transformação de facetas representacionais negativas relacionadas à AIDS e na abordagem da morte como processo natural, ressignificando a própria existência a partir das suas representações, haja vista a função destas como guia para a ação 7,14,21,25.

\section{CONCLUSÃO}

Este estudo buscou descrever os conteúdos e a organização das representações sociais da morte para pessoas que vivem com HIV. Caracterizou-se como provável sistema central para as representações sociais da morte as cognições tristeza e descanso. A dimensão emocional e do conhecimento, presentes em todos os quadrantes, trouxeram à tona representações da morte, apresentando-se como um objeto multifacetado, que por seu caráter reflexivo, traz diversas denominações, ancoradas nos significados construídos historicamente pelos elementos normativos que determinam a tomada de posição deste grupo. As cognições paz, recomeço e aceitação expressam os frutos da finitude da vida para o grupo e estão imbricados na racionalização do processo de morrer. Exprime uma dimensão prática da representação de aceitar o processo futuro da morte em face de sua inevitabilidade. 
O processo de morte e morrer, como outros fenômenos da vida social e pública, podem ser vividos de diferentes formas, de acordo com o momento histórico e os contextos socioculturais. A morte, companheira do homem por todas as fases da vida, se impõe implicada com a forma de ver e viver no mundo. E, o mundo das pessoas que vivem com HIV, neste estudo aponta uma nova direção, diante da cronicidade da síndrome, resultando em representações imbricadas no sentido da vida, do viver e do morrer como processo natural.

\section{REFERÊNCIAS}

1. Kovács M J. Representações de morte. In: Kóvacs MJ, coordenador. Morte e desenvolvimento humano. São Paulo: Casa do Psicólogo; 1992. p.1-13

2. Moreira V, Bloc L, Rocha M. Meanings of finitude in the lived world of people with HIV/AIDS: a phenomenological study. Estud. pesqui. psicol. [Internet] 2012 [cited 2018 Feb 07]; 12(2): 554-71. Available from: https://www.epublicacoes.uerj.br/index.php/revispsi/article/view/8282/6048

3. Oliveira DC. Construction and transformation of social representations of AIDS and implications for health care. Rev. latinoam. enferm. (Online) 2013; [cited 2018 Feb 07]; 21(sp):276-86. Available from: http://www.scielo.br/pdf/rlae/v21nspe/34.pdf

4. Medeiros ARC, Lima RLFC, Medeiros LB, Moraes RM, Vianna RPT. Analysis of surviving of people living with hiv / aids.. Rev. enferm. UFPE on line. 2017. [cited 2018 Feb 07]; 11(1):47-56. Available from: DOI: https://doi.org/10.5205/1981-8963v11i1a11877p47-56-2017

5. Ministério da Saúde (Br). Departamento de DST, Aids e Hepatites Virais, Secretaria de Vigilância em Saúde. Boletim Epidemiológico HIV/AIDS - 2015. [cited 2018 Feb 07]. Available from: http://www.aids.gov.br/pt-br/pub/2015/boletimepidemiologico-hivaids-2015

6. Reis RK, Melo ES, Gir E. Factors associated with inconsistent condom use among people living with HIV/Aids. Rev. bras. enferm. [Online] 2016 [cited 2018 Feb 07]; 69(1):40-6. Available from: http://www.scielo.br/pdf/reben/v69n1/en_0034-7167-reben-6901-0047.pdf

7. Costa TL, Oliveira DC, Formozo GA. Quality of life and AIDS from the perspective of persons living with HIV: a preliminary contribution by the structural approach to social representations. Cad Saúde Pública. [Online] 2015[cited 2018 Feb 07]; 31(2): 365-76. Available from: http://www.scielo.br/pdf/csp/v31n2/0102-311X-csp-31-02-00365.pdf

8. Custódio EM. Maria Julia Kovacs: a researcher reflecting upon death. Bol. Acad. Paul. Psicol. [Online] 2013 [cited 2018 Feb 07]; 33(85), 243-5. Available from: http://pepsic.bvsalud.org/pdf/bapp/v33n85/a03.pdf

9. Ariès P. O homem diante da morte. Tradução de L. Ribeiro. São Paulo: UNESP; 2014.

10. Kovács MJ. Towards death with dignity in the XXI Century. Rev. Bioét. [Internet] 2014[cited 2018 Feb 07]; 22(1): 94-104. Available from: http://www.scielo.br/pdf/bioet/v22n1/a11v22n1.pdf

11. Mota MS, Gomes GC, Coelho MF, Lunardi Filho WD, Sousa LD. Reactions and feelings of nursing professionals facing death of patients under their care. Rev. gaúch. enferm. [Internet] 2011 [cited 2018 Feb 07]; 32(1):129-35. DOI: http://dx.doi.org/10.1590/S1983-14472011000100017

12. Silva MJP. No caminho: fragmentos para ser melhor. São Paulo: Edições Loyola; 2016.

13. Ministério da Saúde (Br). Departamento de DST, Aids e Hepatites Virais. Relatório do Unaids sobre o 90-90-90 [Internet]. Brasília (DF): Ministério da Saúde; 2016. [cited 2018 Feb 07]. Available from: https://unaids.org.br/tag/90-90-90/page/2/

14. Ministério da Saúde (Br). Departamento de DST, Aids e Hepatites Virais. Diretrizes para a organização da rede de profilaxia antirretroviral pós-exposição de risco à infecção pelo HIV - PEP [Internet]. Brasília (DF): Ministério da Saúde; 2016. [cited 2018 Feb 07]. Available from: http://www.aids.gov.br/pt-br/pub/2016/diretrizes-para-organizacao-da-rede-de-profilaxiaantirretroviral-pos-exposicao-de-risco

15. Santos EI, Gomes AMT, Oliveira DC. Representations of vulnerability and empowerment of nurses in the context of HIV/AIDS. Texto \& contexto enferm. (Online) 2014 [cited 2019 Feb 07]; 23:408-16. DOI: http://dx.doi.org/10.1590/010407072014000700013

16. Oliveira DC, Sá CP, Espírito Santo CC, Gonçalves TC, Gomes AMT. Memories and social representations of SUS users about the public health systems. Rev. eletrônica enferm. 2011 [cited 2018 Feb 07];13(1): 30-41. DOI: http://dx.doi.org/10.5216/ree.v13i1.8981

17. Martinez EA, Souza SR, Tocantins FR. Contribution of social representations to health and nursing research. Invest. Educ. enferm. [Internet] 2012 [cited 2018 Feb 07]; 30(1):101-7. Available from: http://www.scielo.org.co/pdf/iee/v30n1/v30n1a12.pdf

18. Sousa LMS, Silva LS, Palmeira AT. Social representations of short distance truck drivers about HIV/AIDS. Psicol. Soc. [Internet] 2014 [cited 2018 Feb 07]; 26(2): 346-55. http://www.scielo.br/pdf/psoc/v26n2/a11v26n2.pdf

19. Oliva LC. A existência e a morte. São Paulo: Martins Fontes; 2012.

20. Kubler-Ross E. Sobre a morte e o morrer: o que os doentes têm pra ensinar a médicos, enfermeiras, religiosos e aos seus próprios parentes. Tradução de P. Menezes. 9a ed. São Paulo: Martins Fontes; 2008.

21. Negrini M. The meaning of death: a look into the human finitude. Soc. Hum. UFSM, 2014 [cited 2018 Feb 07]; 27(1):29-36. Available from: https://periodicos.ufsm.br/sociaisehumanas/article/view/6592/pdf

22. Joint United Nations Programme on HIV/AIDS. Global AIDS update 2016 [Internet]. Geneva (Swi): Joint United Nations Programme on HIV/AIDS; 2016. [cited 2018 Feb 07]. Available from: http://www.unaids.org/sites/default/files/media_asset/global-AIDS-update-2016_en.pdf 
23. Oliveira, SCF, Araújo LA. The finite from the perspective of elderly males: a study of social representations. Rev. Kairós. [Internet]. 2012 [cited 2018 Feb 07]; 15(4): 66-83. Available from: https://revistas.pucsp.br/kairos/article/view/5547/12633

24. Barbosa AGC, Massaroni L, Lima EFA. Meaning of the process of dying and death for multiprofessional staff. J. Res.: fundam. care. online. [Internet] 2016; [cited 2018 Feb 07]; 8(2):4510-7. DOI: http://dx.doi.org/10.9789/2175-5361.2016.v8i2.4510-4517

25. Machado RS, Lima LAA, Silva GRF, Monteiro CFS, Rocha SS. Finitude and death in Western society: a reflection focusing on health professionals. Cult. cuid. [Internet] 2016 [cited 2018 Feb 07]; 20(45):91-7. Available from: https://rua.ua.es/dspace/bitstream/10045/57355/1/CultCuid_45_10.pdf 